\title{
Schoolbus driver performance can be improved with driver training, safety incentivisation, and vehicle roadworthy modifications
}

\author{
A van Niekerk, ${ }^{1,2} \mathrm{PhD} ; \mathbf{R}$ Govender, ${ }^{1,2,3} \mathrm{PhD} ; \mathbf{R}$ Jacobs, ${ }^{1,2} \mathrm{MA} ; \mathbf{A}$ B van As, ${ }^{4} \mathrm{MB}$ ChB, MMed, MBA, FCS, PhD \\ ${ }^{1}$ Violence, Injury and Peace Research Unit, South African Medical Research Council and University of South Africa, Cape Town, \\ South Africa \\ ${ }^{2}$ Institute for Social and Health Sciences, University of South Africa, Cape Town, South Africa \\ ${ }^{3}$ Department of Sociology, Faculty of Humanities, University of Cape Town, South Africa \\ ${ }^{4}$ Childsafe, Cape Town; and Trauma Unit, Red Cross War Memorial Children's Hospital and Division of Paediatric Surgery, \\ Faculty of Health Sciences, University of Cape Town, South Africa
}

Corresponding author: A van Niekerk (ashley.vanniekerk@mrc.ac.za)

In South Africa (SA), the school transport industry provides millions of children with a means of travelling to and from school. The industry has, however, been reported to be plagued by widespread safety concerns. The consequent road traffic incidents have often been attributed to driver factors, including driving in excess of legal speeds or at inappropriate speeds; driving while under the influence of alcohol, while sleepy or fatigued; or driving without using protective equipment for vehicle occupants. There are currently very few SA interventions that specifically target this important industry role-player. The Safe Travel to School Programme was recently implemented by a national child safety agency, with a focus on driver road safety awareness, defensive driver training, eye-testing, vehicle roadworthy inspections with selected upgrades, incentives for safe performance, and implementation of a vehicle telematics tracking system with regular, individual driving behaviour information updates. This quasi-experimental study offers an evaluation of the initial impact on safety performance of this telematics-based driver and vehicle safety intervention in terms of speeding, acceleration, braking, cornering, and time-of-day driving, and compares the school transport driver performance with that of general motorists. Despite concerns that some school transport vehicles are used for multiple purposes outside of school transport duties, at night, and for longer distances, overall these vehicles recorded lower percentages of speeding, lower harsh braking, and lower average harsh cornering and acceleration than general drivers.

S Afr Med J 2017;107(3):188-191. DOI:10.7196/SAMJ.2017.v107i3.12363

\section{The United Nations Decade of Action for Road Safety under threat in South Africa}

The United Nations (UN) Decade of Action for Road Safety 2011 2020 has identified road traffic crashes as a leading cause of death worldwide, with road traffic injuries cited among the three foremost causes of death for people between 5 and 44 years of age. South Africa (SA)'s road traffic mortality rate, currently at 36.1/100 000, has remained at high levels for the last decade, with no significant reduction. ${ }^{[1]}$ More than 14000 fatalities per year occur owing to road traffic accidents, with a further 7500 people left permanently disabled. ${ }^{[2]}$ Pedestrians account for the largest percentage of traffic-related deaths (37.6\%), followed by vehicle passengers (32.7\%). ${ }^{[2]}$ In SA, these widespread passenger casualties, many involving young passengers, are attributed to combinations of infrastructure problems, poor transport systems, unroadworthy school transport vehicles (i.e. school buses and minibuses), and unpredictable driver behaviour. There is significant international evidence of the role and impact of these factors, e.g. half or more of vehicles in low-income and middle-income countries may lack functioning seat belts. ${ }^{[3]}$ Driver factors that have been highlighted include driving in excess of legal or safe speeds, driving while under the influence of alcohol or while fatigued, or driving without passenger protective gear, such as seat belts, child restraints and helmets. All of these factors are highly indicated as major contributors to road crashes, deaths and serious injuries. ${ }^{[4]}$

\section{The minibus industry and scholar transportation}

The minibus industry in SA has emerged as a major public transport role-player and a significant component of school transport. This is especially true for learners from under-resourced communities, who may reside far from schools and consequently have to travel great distances to access their education. Despite the reliance of learners on minibus transportation, the industry has often been criticised for using substandard vehicles, for overloading, and for high-risk driving behaviour, such as speeding. ${ }^{[5]}$ Commuters in under-resourced communities, who are often most dependent on such transportation, are therefore at an increased risk of injury and mortality. Estimates for the number of collisions per vehicle type per 100 million kilometres travelled include a staggering 1106 collisions for minibus taxis, followed by 916 for passenger vehicles, and 571 for buses. ${ }^{[5]}$ These lend considerable support to greater focus and effort being directed towards these vehicles and their drivers.

The research on school transport drivers' knowledge, attitudes and behaviours towards road safety is quite limited. General driver research indicates that only $8 \%$ of them were found to have sufficient knowledge to drive cars, suggesting improvements to the quality of driver training courses. ${ }^{[6]}$ In terms of driver attitude, factors such as drunk driving, compliance with traffic rules, driving a technically unacceptable vehicle, driving experience, and use of seat belts have a significant impact on incidents and injuries. ${ }^{[7]}$ Even though the 
majority of drivers (90\%) recognise the importance of seat belt use, a significant proportion (17-27\%) don't use seat belts. ${ }^{[6]}$ Moreover, although half of drivers report compliance with seat belt use, $40 \%$ considered it irrelevant should they drive cautiously, and $17 \%$ felt it hindered their safety in extreme situations, such as during a hijacking or an accident. Interestingly, drivers with extensive driving experience were more likely to wear seat belts. ${ }^{[6]}$ Other common driver violations include talking on a phone while driving, with $71 \%$ of drivers admitting to responding to calls as often as 8.5 times per day. Worryingly, the majority of such violations are by those responsible for the largest passenger loads, i.e. bus drivers. ${ }^{[6]}$

To address these challenges, practical interventions are required, especially to promote the child's safe travel to and from school in SA and further afield. These include the protection of vehicle occupants by means of functioning seat belts and use thereof, airbag use and adequate passenger compartment design. ${ }^{[3,8]}$ In addition to the protection of vehicle occupants, there is a need for advocacy for greater compliance with road safety rules. ${ }^{[7]}$ The enforcement of compliance is considered key to deterring major contributors to road crashes, deaths and serious injuries; however, laws alone are insufficient to encourage appropriate behaviourial compliance.

\section{Evaluation of an emerging Safe Travel to School Programme}

Research on the school transport industry in SA is sparse. A national child safety agency in 2014 implemented the Safe Travel to School Programme in partnership with a national medical insurance company, with the overall objective of making a contribution to safer minibus school travel for children. The Safe Travel to School Programme sought to stimulate better driver safety performance and compliance with road safety practices through greater road safety awareness, defensive driver training, eye-testing, vehicle roadworthy inspections with selected upgrades, incentives for safe performance, and implementation of a vehicle telematics tracking system with regular, individual driving behaviour information updates. The vehicle tracking system and devices were supplied by the national insurance company, which uses it to monitor and reward good driving behaviour by clients of their vehicle insurance policies. The current study provided an evaluation of this intervention on school transport driver safety behaviour. The specific aims and objectives of the study were:

- To evaluate Safe Travel to School Programme driver safety performance over time in terms of speeding, acceleration, braking, cornering, and time-of-day driving.

- To compare Safe Travel to School Programme driver safety performance with general motorist performance on same metrics.

\section{Methods}

This evaluation comprised two main components. Firstly, a descriptive trend analysis is provided of the safety behaviour of the initial cohort of school transport drivers, from January 2014 to January 2015, with descriptive data obtained from the vehicle tracking system. Secondly, a quasi-experimental, non-equivalent group design was employed wherein the driving performance of school transport drivers was compared with that of general motorists from September 2014 to January 2015 (Fig. 1).

The study population comprised school transport drivers recruited for the Safe Travel to School Programme. Participants were purposively recruited from three of the main public transport hubs in Cape Town, specifically Athlone, Bellville, and Cape Town Central. The telematics devices were installed in their vehicles. Drivers from

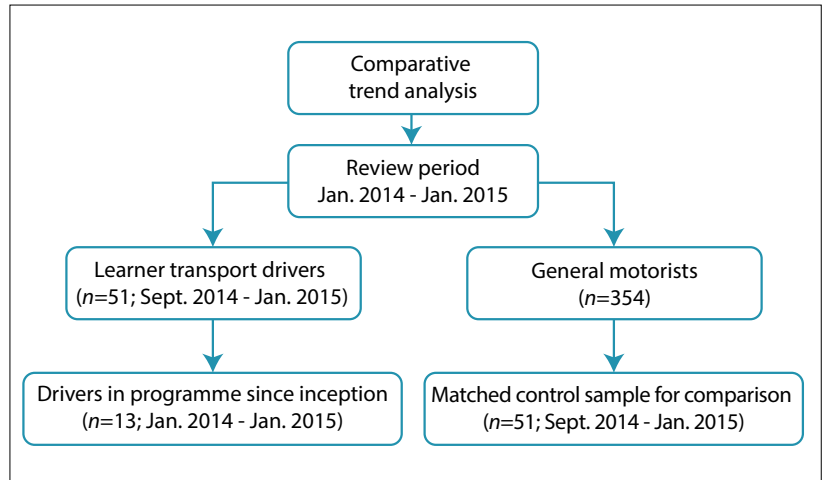

Fig. 1. Comparative trend analysis.

the general motorist group were selected from the national medical insurance company's register of Cape Town drivers, using the same telematics devices, which were matched for age and gender to the Safe Travel to School Programme drivers. For the two evaluation analyses driver safety performance was assessed over time in terms of speeding in excess of $10 \%$ of the speed limit, acceleration, braking and cornering above designated g-force thresholds, and time of day or night driving. Data for these variables were extracted from the vehicle tracking devices.

\section{Data analysis}

Driver safety performance: Vehicle tracking device data

Trend and comparative analyses were conducted using SPSS Version 22 (IBM Corp., USA) software (e.g. inferential $t$-tests, $p<0.05$ ) to identify emerging patterns and trends in driver safety performance behaviours across the variables of interest. As only 13 drivers were identified from January 2014 to January 2015, descriptive statistics were used to examine their driving characteristics with the data obtained from the DQ devices (Discovery, SA). This analysis was expanded from September 2014 to January 2015, for which data were available for 51 school transport drivers. The performance of the Safe Travel to School Programme drivers was then compared with that of 51 general drivers, matched for age and gender, and sourced from the national insurance company's database.

\section{Results \\ School transport driver safety performance: January 2014 - January 2015}

The analysis of speeding, acceleration, braking, cornering and night driving performance suggests that the majority of Safe Travel to School Programme drivers were performing in an appropriate, safe manner, i.e. below the designated threshold for each metric. There were some exceptions observed, which may reflect: (i) habitual serial offenders for the review period; or alternatively (ii) use of the vehicle by more than one driver, therefore accounting for different driving conditions and multiple driver performance styles. Habitual offending among the drivers was not limited to speeding and was also observed for accelerations, braking and cornering. However, observed trends in individual performance were confounded in some instances by excessive night-time use of vehicles, as it is difficult - if not impossible - to differentiate driving performance of school transport drivers during school runs from other forms of driving utilising the same vehicle outside the school transport hours and with different passengers. This confound is a consequence of the telematics device recording driving behaviour of a specific vehicle rather than that of a specific driver. 
Age

In general, the evaluation indicated that school transport drivers aged $\leq 35$ years exceed the speed limit (by 10\%) four times more often than drivers $>35$ years. This study therefore suggests that the different age groups, i.e. $\leq 30 ; 31$ - 44 ; and $\geq 45$ years, would appear to have different safety and performance profiles and driving styles, of which age is arguably only one indicator.

\section{Safety performance of Safe Travel to School Programme drivers compared with insurance company policy holder drivers}

When comparing the Safe Travel to School Programme drivers with the insurance company policy holder drivers from September 2014 to January 2015, it was found that the programme drivers performed significantly better $(t=3.28, p<0.01)$ on the percentage of time at which their speed exceeded the speed limit by $10 \%(n=102)$. With the exception of January 2015 (a vacation period) travel, the programme drivers were found to speed less often (Fig. 2) than the policy holder drivers. As speed is positively correlated with unsafe driving behaviours, the reduced speed for programme drivers could be argued to translate into safer driving behaviours compared with the comparison group.

The programme drivers also performed better than general drivers in terms of their recorded accelerations (Fig. 3). However, these differences were not statistically significant $(t=1.84, p>0.05)$.

Driver braking above a set $\mathrm{g}$-force threshold was higher in September and October 2014, but lower in November - January 2015 (Fig. 4). The reasons for the notable decline in braking above the g-force threshold between October and September 2014 are unknown, as data on the implementation of the individual Safe Travel to School Programme interventions were not available to test possible effects on driving performance. However, the lower levels observed from November 2014 to January 2015 could be explained by the likely long-distance travelling on vacation routes, for which braking is required less often than when driving in urban and high-density areas.

Safer driving performance of Safe Travel to School Programme drivers was also reflected in the average monthly cornering above the designated g-force threshold (Fig. 5). These noticeable differences between the programme and general drivers could nevertheless be explained by differences in cornering thresholds for vehicle type, i.e. sedans compared with minibus vehicles. Benchmarking for specific vehicle types is required for more meaningful comparisons.

On average, the programme drivers were found to engage in more night driving during weekdays and weekends than the comparison group. The marginally higher rates of speeding of $>10 \%$ above the speed limit of drivers undertaking night drives suggests: (i) qualitative differences in driving conditions during this period compared with daytime driving; and/or (ii) different drivers for the same vehicle for daytime compared with night-time driving.

In summary, although the Safe Travel to School Programme vehicles may well have been used outside of school transport duties, at night and for longer distances, overall the vehicles participating in the safety programme recorded lower percentages of time speeding, lower harsh braking, and lower average harsh cornering and acceleration than general drivers.

\section{Conclusions and recommendations}

The results of this first evaluation of the Safe Travel to School Programme are promising. School transport drivers appear to perform relatively better than general motorists with regard to key driving performance indicators (speeding, acceleration, braking and cornering) for the particular review period.

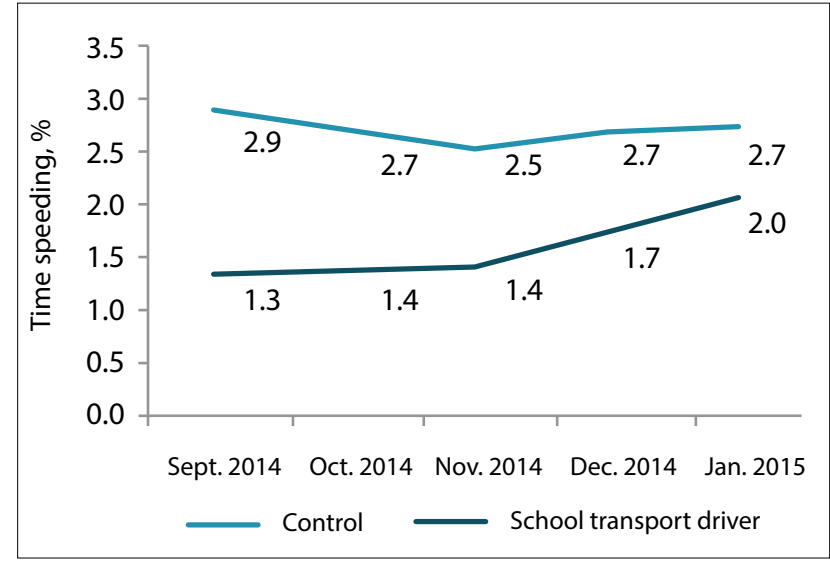

Fig. 2. Percentage of time that speed exceeded $10 \%$ of the limit $(\mathrm{n}=102)$.

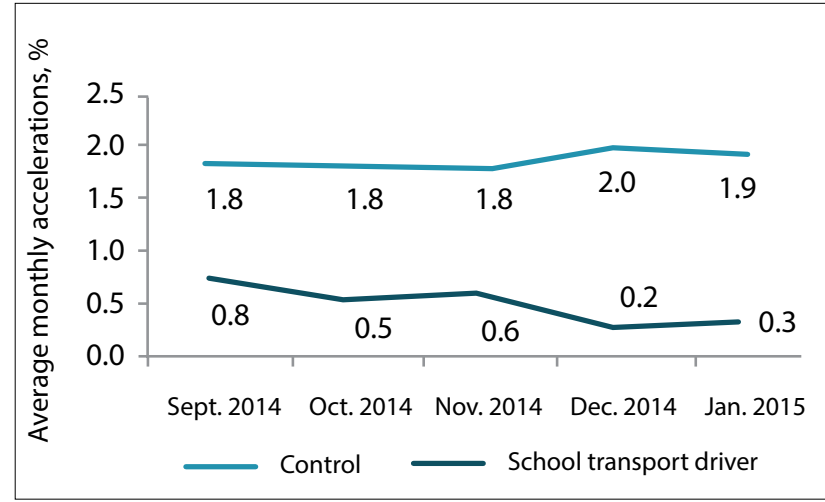

Fig. 3. Average monthly accelerations above g-force threshold $(\mathrm{n}=102)$.

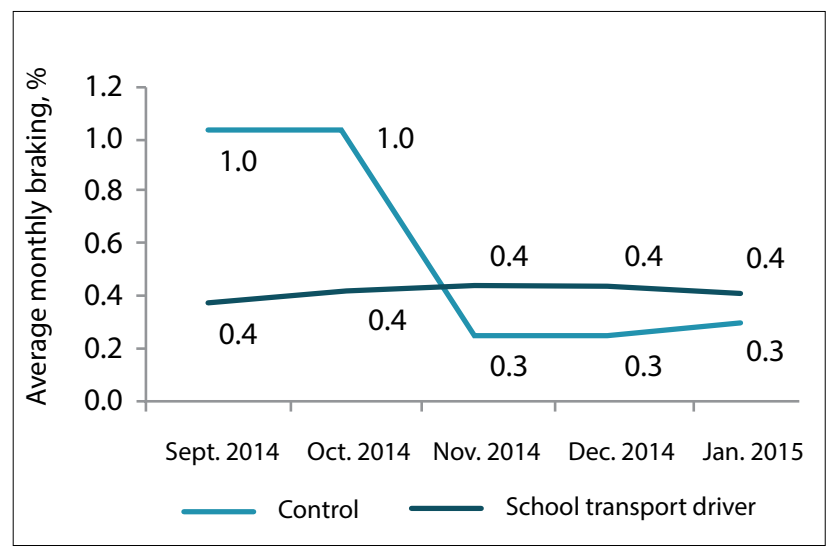

Fig. 4. Average monthly braking above $\mathrm{g}$-force threshold $(\mathrm{n}=102)$.

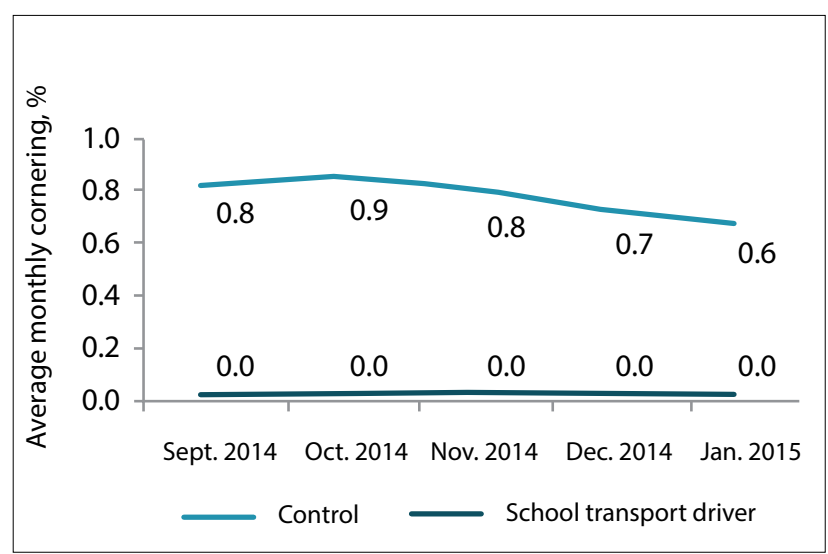

Fig. 5. Average monthly cornering above g-force threshold $(\mathrm{n}=102)$. 


\section{Driver demographics}

Regardless of the differences in driver behaviours, age and gender (male $)^{[9-11]}$ are highly significant contributors to unsafe driving behaviours and should be considered and accounted for in the design of driver safety interventions. Further interventions should consider a staggered programme that takes driver age (at least differentiated for $<35$ years), but preferably the different capacities, experiences and needs of the $\leq 30,31-44$, and $\geq 45$-year groups into account. There is, however, a need to collect information relevant to each driver, beyond demographic information, e.g. driver history and psychosocial characteristics. This information is critical for two broad reasons: (i) to provide better contextual information for the analysis and explanation of individual driving performance; and (ii) to ensure a better fit of designed safety interventions to specific drivers or cohorts of drivers.

\section{Persistent defaulters}

This study has implications for other interventions that may be considered in this sector. For instance, there is good reason to suggest remedial action interventions based on observed performance of drivers and identified lapses in specific driving behaviours. This is essential to mitigate the prolonged impact of repeat or serious road traffic offenders on such driver programmes and on the learners being transported in vehicles, who are part of the programme. The purpose of such a remedial component would be to identify such participants timeously and intentionally modify their driving behaviours to ensure their continued participation in the programme. Participants who fail to respond to such remedial action should be removed from the programme to minimise the potential of likely harm to learners and to limit legal and other liability for programme partners and sponsors.

\section{Sustainable long-term intervention impacts}

The Safe Travel to School Programme applied an incentive-based token economy system, which may not be sustainable for long-term behavioural modification, especially in resource-strapped settings. In general, such a system is known to produce changes in behaviour that are transient or easily altered, given the absence of the token economy. ${ }^{[12]}$ Therefore, incentives should be awarded on good or improved driving behaviours over sustained periods of time. More cogently, long-term behaviour modification is best obtained through internalisation of relevant attitudes and behaviours by drivers themselves, and the token economy is therefore most appropriately utilised to augment and not replace this aspect of behavioural change.

\section{Road safety and child injury prevention}

The findings of this study reflect the need for further holistic evaluations of driver performance, interventions directed at driver health, safety and wellbeing (in a competitive, unprotected industry), and rigorous screening of potential drivers. ${ }^{[13]}$ Children are safer with older, more experienced drivers. It is, however, the collective social responsibility of the transportation sector, parents, broader communities, and child safety practitioners to mobilise and support efforts to enable the rigorous screening, training and remediation of school transport drivers.

The SA government has recognised the challenges faced with the transportation of learners to and from schools, and has drafted the National Learner Transport Policy in collaboration with the Department of Basic Education and other stakeholders with the aim to fulfil the constitutional mandate to provide safe and efficient transportation for learners. ${ }^{[14]}$ However, the implementation of this policy across privately dedicated school transport operations has proved to be challenging, as critiques lodged at this policy demand guidelines and firmer regulation of the industry to ensure that every child benefits from the available, although restrictive, funding structures (i.e. subsidies), ${ }^{[15]}$ and a broader social responsibility for child safety on our roads.

Acknowledgements. This project is a Childsafe South Africa project, which has been financially supported by the Discovery Trust. The authors acknowledge the Discovery Foundation and Discovery Insure for their ongoing support during the project.

Matzopoulos R, Prinsloo M, Pillay-van Wyk V, et al. Injury-related mortality in South Africa: A retrospective descriptive study of postmortem investigations. Bull World Health Organ 015:93(5):303-313. http://dx.doi.org/10.2471/blt.14.14577

2. Road Traffic Management Corporation. Road Traffic Report: Calendar 1 January - 31 December 2015. Pretoria: RTMC, 2016. http://www.rtmc.co.za/index.php/reports/traffic-reports (accessed 20 January 2017). 3. Forjuoh SN. Traffic-related injury prevention interventions for low-income countries. Int J Injury Control Safety Promotion 2003;10(1-2):109-118. http://dx.doi.org/10.1076/icsp.10.1.109.14115

4. European Transport Safety Council. Police Enforcement Strategies to Reduce Traffic Casualties in Europe. Brussels: ETSC, 1999

5. Sukhai A, Noah M, Prinsloo M. Road traffic injury in South Africa: An epidemiological overview for 2001. In: Suffla S, van Niekerk A, Duncan N, eds. Crime, Violence and Injury Prevention in South Africa: 6. Developments and Challenges. Tygerberg South African Medical Research Council 2004:114-127.

6. Demberelsuren J. Knowledge, Attitudes and Practices of Pedestrians, Drivers and Traffic Policemen on . Demberelsuren J. Knowledge, Attitudes and Practices of Pedestrians, Drivers and
Traffic Safety Related Issues. Mongolia: Millennium Challenge Corporation, 2010.

7. World Health Organization. World Report on Road Traffic Injury Prevention: Summary. Geneva: WHO, 2004

8ummings P, McKnight B, Rivara FP, Grossman DC. Association of driver air bags with driver fatality: A matched cohort study. BMJ 2002;324(7346):1119-1122. http://dx.doi.org/10.1136/bmj.324.7346.1119 9. Rhodes N, Pivik K. Age and gender differences in risky driving: The roles of positive affect and risk perception. Accident Anal Prevent 2011;43(3):923-931. http://dx.doi.org/10.1016/j.aap.2010.11.015

10. Tabibi Z, Borzabadi HH, Stavrinos D, Mashhadi A. Predicting aberrant driving behaviour: The role of executive function. Transportation Research Part F. Traffic Psychol Behav 2015;34:18-28. http://dx.doi. org/10.1016/.trf 2015.07 .015

11. Cheng ASK, Lee HC. Risk-taking behavior and response inhibition of commuter motorcyclists with different levels of impulsivity. Transportation Research Part F. Traffic Psychol Behav 2012;15(5):535-543. http://dx.doi. org $/ 10.1016 / j$ trf 2012.05 .005

12. Levine FM, Fasnacht G. Token rewards may lead to token learning. Am Psychol 1974;29(11):816-820. http://dx.doi.org/10.1037/h0037474

13. Machin MA, de Souza JMD. Predicting health outcomes and safety behaviour in taxi drivers. Transportation Research Part F. Traffic Psychol Behav 2004; 7(4-5):257-270. http://dx.doi.org/10.1016/j.trf.2004.09.004

14. Department of Transport. National Learner Transport Policy. Pretoria: DoT, 2015. http://www.gov.za/sites/ www.gov.za/files/39314_gon997.pdf (accessed 27 May 2016).

15. Essop R. Opposition hits out at learner transport policy. Eyewitness News, 2015. http://ewn.co.za/2015/06/01/ Opposition-hits-out-at-learner-transport-policy (accessed 19 May 2016). 